EPJ Web of Conferences 66, 06022 (2014)

DOI: $10.1051 /$ epjconf/ 20146606022

(C) Owned by the authors, published by EDP Sciences, 2014

\title{
Short-range correlations of partons \& 3D nucleon structure
}

\author{
P. Schweitzer ${ }^{1}$ \\ ${ }^{1}$ Department of Physics, University of Connecticut, Storrs, CT 06269, USA
}

\begin{abstract}
Dynamical breaking of chiral symmetry in QCD is caused by nonperturbative interactions on a scale $\rho \sim 0.3 \mathrm{fm}$ much smaller than the hadronic size $R \sim 1 \mathrm{fm}$. This has important consequences for the nucleon structure such as the prediction that the transverse momentum distribution of sea quarks is significantly broader than the $p_{T}$-distribution of valence quarks due to short-range correlations between sea quarks in the nucleon's light-cone wave function.
\end{abstract}

\section{Introduction}

Our knowledge of the internal quark-gluon structure of the nucleon is due to studies of inclusive lepton-nucleon deep-inelastic scattering (DIS), Drell-Yan processes and other reactions that are described in terms of universal, process-independent parton distribution functions. In the infinite momentum frame parton distribution functions are interpreted as probabilities to find partons of the flavor $a=u, d, \bar{u}, \ldots$ carrying the fraction $x$ of the nucleon's momentum at a resolution scale $\mu$ associated with the hard scale of the process.

Transverse momentum dependent distribution functions (TMDs) are a natural extension of this concept [1]. By taking into account the transverse motion of partons and spin-orbit correlations, they provide unique insights in the 3D nucleon structure. TMDs are relevant for the description of transverse (with respect to the hard momentum flow in the process) momenta in the final state, e.g., transverse momenta of hadrons produced in semi-inclusive DIS. On the basis of rigorous factorization theorems which determine their universality and evolution properties [2-6] TMDs constitute powerful tools that allow us to significantly increase the present understanding of the nucleon structure $[7,8]$.

The nonperturbative properties of TMDs are largely unknown. Many data [9-12] are well described assuming Gaussian $p_{T}$-dependence of TMDs $[13,14]$ which should be compatible with high energy data after considering evolution $[15,16]$. Quark models support approximate Gaussian $p_{T}$-behavior of valence quarks [17-20]. But much less is known about sea quarks.

The chiral quark soliton model [21], which was derived from the instanton model of the QCD vacuum [22], is an effective theory which successfully and consistently accounts for sea quark effects [23-30]. The model was recently applied to TMDs [31-33] and yields remarkable predictions for the $3 \mathrm{D}$ nucleon structure at a low scale. The $p_{T}$-behavior of valence quark distributions $(q-\bar{q})$ is governed by the inverse nucleon size $R^{-1} \sim 0.2 \mathrm{GeV}$ and drop steeply at large $p_{T}$. The sea quark distributions $\bar{q}$ are dominated by nonperturbative chiral-symmetry breaking interactions and extend up to the scale $\rho^{-1} \sim 0.6 \mathrm{GeV}$. The new results and their far-reaching phenomenological consequences are briefly reviewed. 


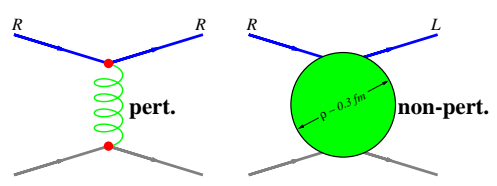

(a)

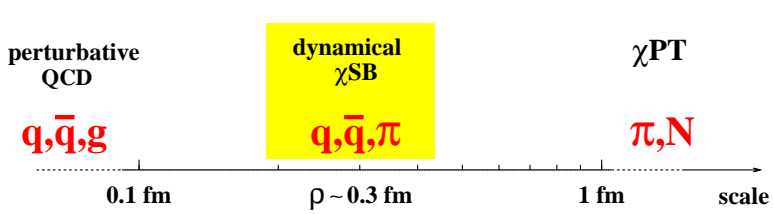

(b)

Figure 1. (a) Perturbative QCD preserves chirality of right-handed (R) or left-handed (L) quarks, but nonperturbative effects can flip it [34]. (b) Effective degrees of freedom of the strong interaction at different length scales. Dynamical chiral symmetry breaking occurs at the scale $\rho$.

\section{Chiral symmetry breaking, and the 2 scale picture}

The dominating feature of strong interactions at low energies is spontaneous chiral symmetry breaking with the emergence of Goldstone bosons (pions) and a quark vacuum condensate [35]. A dynamical mechanism for that is due to nonperturbative QCD interactions caused by topological gauge field configurations which can flip chirality, see Fig. 1a. This picture is realized in the instanton vacuum model [22] where the average instanton size sets the shortdistance nonperturbative scale $\rho \sim 0.3 \mathrm{fm}$, whose existence is supported by independent evidence [36-39]. The degrees of freedom at the scale $\rho$ are Goldstone bosons, and effective $q, \bar{q}$ with a dynamically generated constituent quark mass $M \sim 350 \mathrm{MeV}$, see Fig. 1b.

Thus, hadron structure is determined by the interplay of two nonperturbative scales. The longer distance scale $R \sim 1 / M$ fixes the nucleon size and governs the dynamics of "valence quarks" which is described, under varying assumptions, in quark models. Hence one naturally expects typical transverse momenta of valence quarks to be $\left\langle p_{T}^{2}\right\rangle_{\text {val }} \sim M^{2}$ at low initial scales, and this what quark models yield [17-20]. In contrast to this, the dynamics of sea quarks is governed by the shorter distance scale $\rho$ which is the typical size of configurations in which correlated $\bar{q} q$-pairs are generated nonperturbatively, see Fig. 2. Hence, one expects $\left\langle p_{T}^{2}\right\rangle_{\text {sea }} \sim \rho^{-2}$ and this has recently been observed in the chiral quark soliton model [31-33].

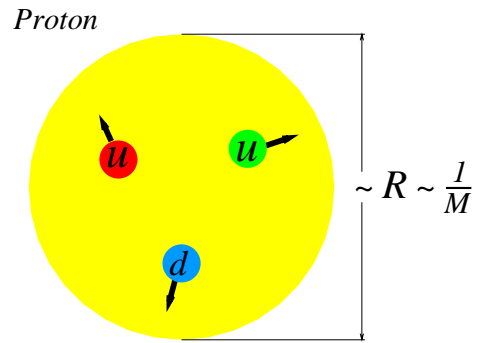

(a)

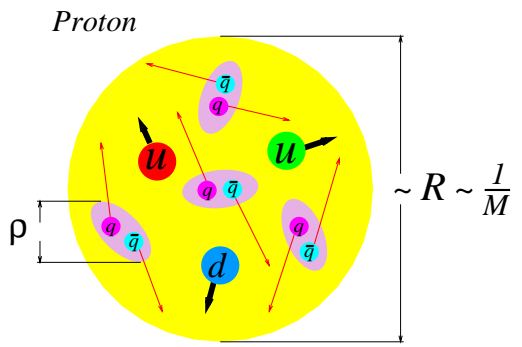

(b)

Figure 2. The two nonperturbative scales governing the structure of the nucleon: $R=1 / M \sim 1 \mathrm{fm}$ which sets the size of the nucleon and describes valence quark effects (a), and $\rho \ll R$ which describes short-range correlations in the nucleon and governs the dynamics of sea quarks (b). 


\section{Chiral quark-soliton model}

The instanton model describes the QCD vacuum as a strongly interacting, dilute ensemble of instantons and anti-instantons in which light quarks acquire a dynamically generated constituent mass $M$ at small Euclidean momenta and chiral symmetry is dynamically broken. The parameter $(M \rho)^{2}$, which is proportional to the instanton packing fraction and is small due to the diluteness of the medium, plays a crucial role in the derivation of this picture [22].

In leading order of the small parameter $(M \rho)^{2}$ the strong interaction is described by the low energy effective theory formulated in terms of $q, \bar{q}, \pi^{a}$ degrees of freedom

$$
\mathcal{L}_{\text {eff }}=\bar{\psi}\left(i \not \partial+M \exp \left(i \gamma_{5} \tau^{a} \pi^{a} / F_{\pi}\right)\right) \psi .
$$

The effective coupling between quarks and Goldstone bosons is large, $M / F_{\pi} \sim 4$, and the theory has to be solved nonperturbatively in a large- $N_{c}$ expansion in which the nucleon emerges as a chiral soliton, i.e. an extended system of quarks and antiquarks bound in a self-consistent background pion field. This is the chiral quarksoliton model ( $\chi \mathrm{QSM})[21]$, which provides a basically parameter-free description of static nucleon observables, form factors, and parton distribution functions

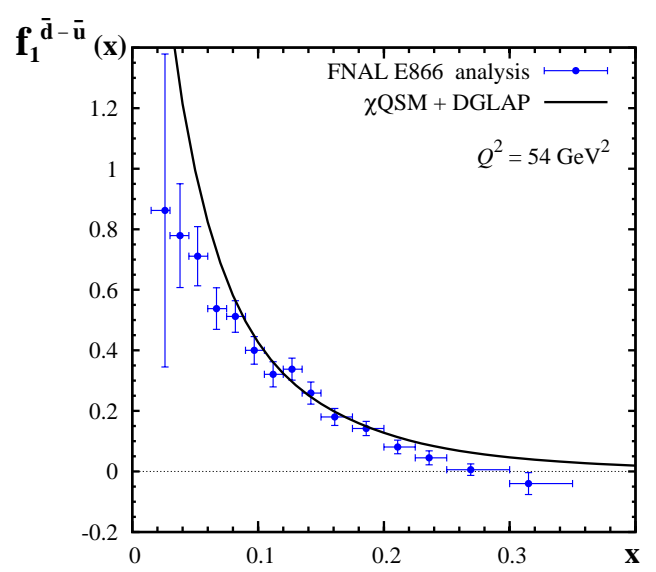

Figure 3. $f_{1}^{\bar{d}-\bar{u}}(x)$ from $\chi$ QSM [26] at the scale $Q^{2}=54 \mathrm{GeV}^{2}$ in comparison to the Fermilab E866 Drell-Yan analysis [40]. [23-25].

Results for parton distribution functions from the model refer to a low scale $\mu \sim 0.6 \mathrm{GeV}$. The precise matching of model $q, \bar{q}$ degrees of freedom and QCD $q, \bar{q}$ degrees of freedom at the scale $\mu \sim 1 / \rho$ is highly non-trivial [24-26]. Flavor-nonsinglet quantities which do not mix with gluons under leading-order evolution are less sensitive to details of the matching procedure. Fig. 3 shows the model result for $f_{1}^{\bar{d}-\bar{u}}(x)$ from [26] evolved to $Q^{2}=54 \mathrm{GeV}^{2}$ which is in very good agreement with the Fermilab E866 Drell-Yan analysis [40]. Typically $\chi$ QSM results are found to agree within (10-30)\% accuracy with phenomenology.

Among the most interesting predictions of the $\chi$ QSM is that there is a large flavor asymmetry also in the helicity sea $[24,25]$ which leads to specific predictions for the Drell-Yan process [27]. Recent global QCD fits [41] and especially preliminary data from RHIC on $W^{ \pm}$ production data [42] support the prediction.

\section{TMDs in the chiral quark-soliton model}

Parton distributions can be obtained in the $\chi$ QSM either starting from (i) QCD definitions as nucleon matrix elements of quark light-ray operators [24], or (ii) their parton model definitions as number densities of particles in a nucleon in infinite momentum frame [25]. Using (ii) as definition for TMDs yields in leading order of the $1 / N_{c}$ expansion [32]

$$
f_{1}^{u+d}\left(x, p_{T}\right)=\frac{M_{N} N_{c}}{(2 \pi)^{3}} \sum_{n \text { occ }} \bar{\phi}_{n}(\vec{p}) \gamma^{+} \phi_{n}(\vec{p}), g_{1}^{u-d}\left(x, p_{T}\right)=\frac{M_{N} N_{c}}{3(2 \pi)^{3}} \sum_{n \text { occ }} \bar{\phi}_{n}(\vec{p}) \gamma_{5} \gamma^{+} \tau^{3} \phi_{n}(\vec{p})
$$


where the sums go over "occupied states" in the spectrum of the effective Hamiltonian, i.e. the bound state and the negative Dirac continuum, and we defined $\gamma^{+}=\gamma^{0}+\gamma^{3}$ and $\vec{p}=\left(\vec{p}_{T}, x M_{N}-E_{n}\right)$. The corresponding sea quark TMDs are given by similar expressions, and other flavor combinations appear only at subleading order in the large- $N_{c}$ expansion.

In Ref. [31] $f_{1}^{u+d}\left(x, p_{T}\right)$ was studied numerically. In [32] $f_{1}^{u+d}\left(x, p_{T}\right)$ and $g_{1}^{u-d}\left(x, p_{T}\right)$ were evaluated analytically using the "interpolation formula" which is based on an expansion in gradients of the chiral field $U=\exp \left(i \pi^{a} \tau^{a} / F_{\pi}\right)$ and provides excellent approximations [24].

Fig. 4 shows a result for $f_{1}^{a}\left(x, p_{T}\right)$ from [32]. Remarkably, valence and sea quark distributions have significantly different $p_{T}$-behavior. At smaller $p_{T}$ valence quarks dominate, at larger $p_{T}$ sea quarks.

This is in line with the expectations that valence quark dynamics is dominated by the scale $R$ such that $\left\langle p_{T}^{2}\right\rangle_{\text {val }} \sim 1 / R^{2}$, while sea quark dynamics is governed by the scale $\rho$ and $\left\langle p_{T}^{2}\right\rangle_{\text {sea }} \sim 1 / \rho^{2}$. Since $\rho \ll R$ one is naturally lead to the expectation, see the discussion in Sec. 2,

$$
\left\langle p_{T}^{2}\right\rangle_{\text {sea }} \gg\left\langle p_{T}^{2}\right\rangle_{\text {val }}
$$

and the model results confirm this. Hereby is understood that the $\left\langle p_{T}^{2}\right\rangle_{i}$ quantify the "slopes" of the respective distributions in Fig. 4 rather than definite nucleon matrix elements of QCDoperators (this points deserves more detailed studies).

The results for $g_{1}^{a}\left(x, p_{T}\right)$ are qualitatively similar. Interestingly, the $p_{T}$-slopes of the sea quark distributions $f_{1}^{\bar{u}+\bar{d}}\left(x, p_{T}\right)$ and $g_{1}^{\bar{u}-\bar{d}}\left(x, p_{T}\right)$ become equal in the region $M \ll p_{T} \ll 1 / \rho$ which can be understood as an instance of "restoration of chiral symmetry" [32].

\section{Phenomenological implications}

The different $p_{T}$-behavior of sea and valence TMDs has interesting phenomenological consequences. The separation of valence- and sea-quark effects is not straightforward though: quarks (valence + sea) or antiquarks (sea) enter in processes, but not valence quarks.

Studies of charged kaon production in DIS at small $x$ and large $z$ could be insightful. In fact, the production of negative kaons $K^{-} \sim \bar{u} s$ is dominated by sea quarks struck from the target (at large- $z$ where favored fragmentation dominates over unfavored fragmentation). In contrast to this, in the production of positive kaons $K^{+} \sim u \bar{s}$ also valence quarks play an important role. Hence, one can expect that the cross section for $K^{+}$production falls off faster with transverse hadron momentum $P_{h \perp}$ than that for $K^{-}$production [32]. This effect could be measured at JLab 12 or a future EIC. Similarly promising insights could be due to combining cross sections for charged pion production [32]. 
INPC 2013

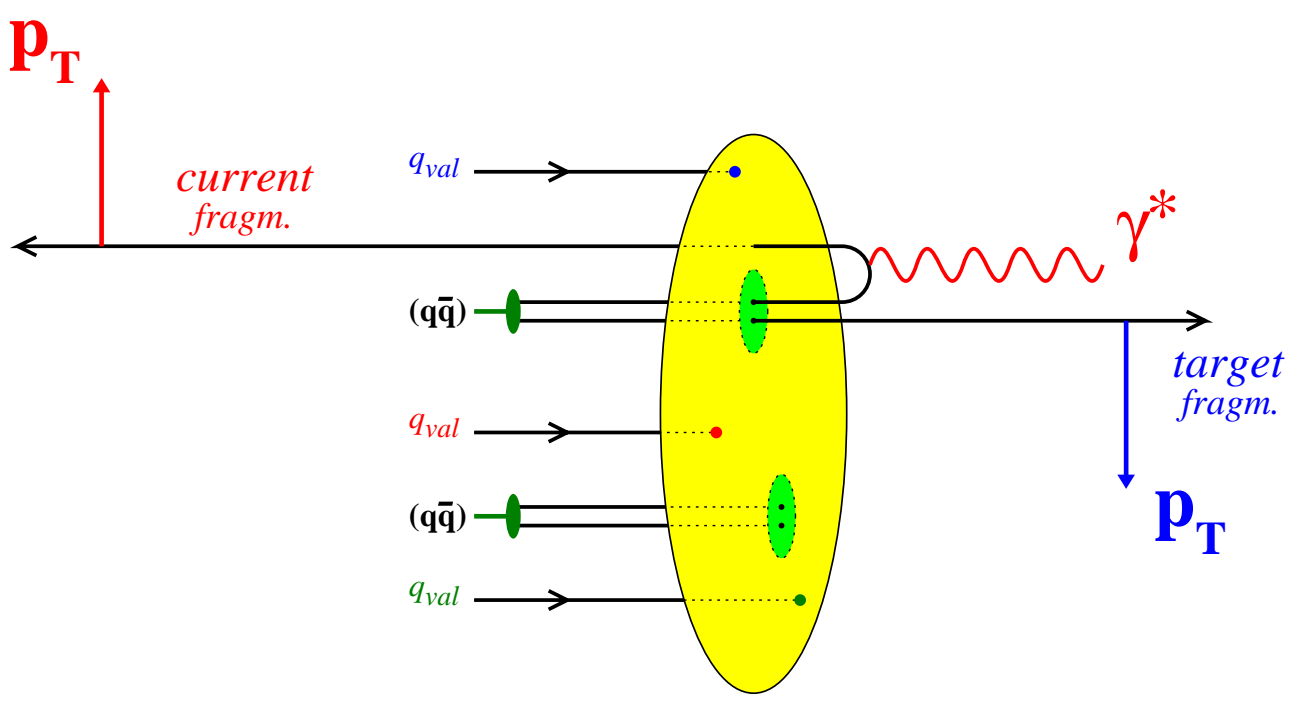

Figure 5. Correlations of transverse hadron momenta in current and target fragmentation regions. When a virtual photon (coming from the right) splits up a correlated $\bar{q} q$ pair, one expects correlations of transverse hadron momenta in the current and target fragmentation regions.

The larger transverse momenta of sea quarks as compared to valence quarks are due to short distance correlations of $\bar{q} q$-pairs sketched in Fig. 2. The quantum numbers of the correlated $\bar{q} q$-pairs are scalar-isoscalar or vector-isovector. In principle it is possible to observe such correlations, which are in analogy to short-range $N N$ correlations in nuclei [43].

For instance, if in semi-inclusive DIS the virtual photon "breaks up" a pair of $\bar{q}, q$ which are strongly correlated at the distance scale $\rho$, then both correlated particles will produce jets (or hadrons) with opposite transverse momenta, see Fig. 5. The fragmentation process tends to dilute the effect, and so does gluon radiation at too large rapidity separations. But under controlled conditions the effect could be promising [32]. The partonic short distance correlations observed in the chiral theory could have also important implications for the understanding of multi-parton correlations which lead to multi-jet events in high-energy reactions [46].

Another interesting question is whether the model results can be used to inspire fit Ansätze for TMDs [6]. Further dedicated studies are needed.

\section{Conclusions}

The unpolarized and helicity TMDs were studied in the $\chi$ QSM. The valence quark results agree qualitatively with other quark models and exhibit a roughly Gaussian $p_{T}$-behavior. For sea quarks the predicted $p_{T}$-behavior is different with $\left\langle p_{T}^{2}\right\rangle_{\text {sea }} \gg\left\langle p_{T}^{2}\right\rangle_{\text {val }}$. This is true for both the unpolarized and the helicity TMDs, $f_{1}^{a}\left(x, p_{T}\right)$ and $g_{1}^{a}\left(x, p_{T}\right)$, but not necessarily for other TMDs [28]. The present data are not sensitive enough [14, 15]. But the prediction could be tested in charged pion or kaon production at JLab12 or a future Electron-Ion Collider [8]. Other interesting implications include transverse momentum correlations between target and current fragmentation regions in DIS. 
While our results signal the importance of chiral symmetry breaking and the QCD vacuum structure for TMDs, also open questions remain [32]. Why do parton model based approaches $[44,47,48]$ yield smaller $\left\langle p_{T}^{2}\right\rangle$ than the TMD approach? Is the contribution from the Wilsonlines in TMDs [1] suppressed by the instanton packing fraction as it is for twist-2 parton distributions [49]? These and further questions will be addressed in future work.

Acknowledgements: This talk reports joint work with Mark Strikman and Christian Weiss to whom the author is indebted for numerous discussions and comments. The work is partially supported by U.S. DOE contract No. DE-AC05-06OR23177 under which Jefferson Science Associates, LLC operates Jefferson Lab.

\section{References}

[1] J. C. Collins, "Foundations of Perturbative QCD" (Cambridge University Press, Cambridge, 2011). J. Collins, arXiv:1107.4123 [hep-ph].

[2] J. C. Collins and D. E. Soper, Nucl. Phys. B 193, 381 (1981); B 213, 545E (1983).

J. C. Collins, D. E. Soper and G. Sterman, Nucl. Phys. B 250, 199 (1985).

[3] X. D. Ji, J. P. Ma, and F. Yuan, Phys. Rev. D 71, 034005 (2005); Phys. Lett. B 597, 299 (2004). J. C. Collins and A. Metz, Phys. Rev. Lett. 93, 252001 (2004).

[4] I. O. Cherednikov and N. G. Stefanis, Nucl. Phys. B 802, 146 (2008).

I. O. Cherednikov, A. I. Karanikas and N. G. Stefanis, Nucl. Phys. B 840, 379 (2010).

[5] M. G. Echevarria, A. Idilbi and I. Scimemi, JHEP 1207, 002 (2012).

[6] J. Collins, arXiv:1307.2920 [hep-ph].

[7] D. W. Sivers, Phys. Rev. D 41, 83 (1990); Phys. Rev. D 43, 261 (1991).

A. Kotzinian, Nucl. Phys. B 441, 234 (1995).

P. J. Mulders and R. D. Tangerman, Nucl. Phys. B 461, 197 (1996); B 484, 538E (1997).

D. Boer and P. J. Mulders, Phys. Rev. D 57, 5780 (1998).

J. C. Collins, Phys. Lett. B 536, 43 (2002).

A. V. Belitsky, X. Ji and F. Yuan, Nucl. Phys. B 656, 165 (2003).

D. Boer, P. J. Mulders and F. Pijlman, Nucl. Phys. B 667, 201 (2003).

S. Arnold, A. Metz, M. Schlegel, Phys. Rev. D79, 034005 (2009).

[8] A. Accardi, J. L. Albacete, M. Anselmino, N. Armesto, E. C. Aschenauer, A. Bacchetta, D. Boer and W. Brooks et al., arXiv:1212.1701 [nucl-ex]. D. Boer, M. Diehl, R. Milner, R. Venugopalan, W. Vogelsang, D. Kaplan, H. Montgomery, S. Vigdor et al., arXiv:1108.1713 [nucl-th]. M. Anselmino et al., Eur. Phys. J. A47, 35 (2011).

[9] M. Osipenko et al. [CLAS Collaboration], Phys. Rev. D 80, 032004 (2009).

[10] H. Mkrtchyan et al. [Hall A Collaboration], Phys. Lett. B 665, 20 (2008).

[11] A. Airapetian et al. [HERMES Collaboration], Phys. Lett. B 684, 114 (2010).

[12] C. Adolph et al. [COMPASS Collaboration], arXiv:1305.7317 [hep-ex].

[13] U. D'Alesio and F. Murgia, Prog. Part. Nucl. Phys. 61, 394 (2008).

[14] P. Schweitzer, T. Teckentrup, A. Metz, Phys. Rev. D81, 094019 (2010).

[15] F. Landry, R. Brock, P. M. Nadolsky, C. P. Yuan, Phys. Rev. D67, 073016 (2003).

[16] S. M. Aybat, T. C. Rogers, Phys. Rev. D83, 114042 (2011).

P. Sun and F. Yuan, Phys. Rev. D 88, 034016 (2013); arXiv:1308.5003 [hep-ph]. 
[17] R. Jakob, P. J. Mulders and J. Rodrigues, Nucl. Phys. A 626, 937 (1997).

L. P. Gamberg, G. R. Goldstein and M. Schlegel, Phys. Rev. D 77, 094016 (2008).

A. Bacchetta, F. Conti and M. Radici, Phys. Rev. D 78, 074010 (2008).

[18] H. Avakian et al, Phys. Rev. D78, 114024 (2008); Phys. Rev. D81, 074035 (2010).

[19] B. Pasquini, S. Cazzaniga and S. Boffi, Phys. Rev. D 78, 034025 (2008).

[20] H. H. Matevosyan, W. Bentz, I. C. Cloet, A. W. Thomas, Phys. Rev. D 85, 014021 (2012).

[21] D. Diakonov, V. Y. Petrov and P. V. Pobylitsa, Nucl. Phys. B 306, 809 (1988).

[22] D. Diakonov, V. Y. Petrov, Nucl. Phys. B272, 457 (1986).

[23] C. V. Christov et al, Prog. Part. Nucl. Phys. 37, 91 (1996).

[24] D. Diakonov, V. Petrov, P. Pobylitsa, M. Polyakov, C. Weiss, Nucl. Phys. B 480, 341 (1996).

[25] D. Diakonov, V. Petrov, P. Pobylitsa, M. Polyakov, C. Weiss, Phys. Rev. D 56, 4069 (1997).

[26] P. V. Pobylitsa, M. V. Polyakov, K. Goeke, T. Watabe, C. Weiss, Phys. Rev. D59, 034024 (1999).

[27] B. Dressler et al, Eur. Phys. J. C18, 719-722 (2001).

[28] P. Schweitzer, D. Urbano, M. V. Polyakov, C. Weiss, P. V. Pobylitsa and K. Goeke, Phys. Rev. D 64 (2001) 034013. P. Schweitzer, Phys. Rev. D67, 114010 (2003).

[29] M. Wakamatsu, Phys. Rev. D67, 034006 (2003).

[30] K. Goeke et al, Phys. Rev. D75, 094021 (2007); Phys. Rev. C75, 055207 (2007).

[31] M. Wakamatsu, Phys. Rev. D79, 094028 (2009).

[32] P. Schweitzer, M. Strikman and C. Weiss, JHEP 1301, 163 (2013).

[33] P. Schweitzer, M. Strikman and C. Weiss, Acta Phys. Polon. Supp. 6, 109 (2013); and arXiv:1309.2990 [hep-ph].

[34] D. Diakonov, Prog. Part. Nucl. Phys. 51, 173 (2003).

[35] J. Gasser, H. Leutwyler, Phys. Rept. 87, 77 (1982).

[36] M. V. Polyakov and C. Weiss, Phys. Lett. B 387, 841 (1996).

[37] D. Diakonov, Prog. Part. Nucl. Phys. 36, 1 (1996).

[38] C. D. Roberts, arXiv:1203.5341 [nucl-th].

[39] D. Ebert and M. K. Volkov, Z. Phys. C 16, 205 (1983).

[40] R. S. Towell et al. [FNAL E866/NuSea Collaboration], Phys. Rev. D 64, 052002 (2001).

[41] D. de Florian, R. Sassot, M. Stratmann and W. Vogelsang, Phys. Rev. Lett. 101, 072001 (2008).

[42] J. R. Stevens [ STAR Collaboration], arXiv:1302.6639 [nucl-ex].

[43] For a review, see: J. Arrington, D. W. Higinbotham, G. Rosner and M. Sargsian, Prog. Part. Nucl. Phys. 67, 898 (2012).

[44] A. V. Efremov et al, Phys. Rev. D80, 014021 (2009); Phys. Rev. D 83, 054025 (2011).

[45] C. Bourrely, F. Buccella and J. Soffer, Int. J. Mod. Phys. A 28, 1350026 (2013); Mod. Phys. Lett. A 21, 143 (2006)

[46] B. Blok, Y. Dokshitzer, L. Frankfurt and M. Strikman, arXiv:1306.3763 [hep-ph].

[47] R. K. Ellis, W. Furmanski, R. Petronzio, Nucl. Phys. B207, 1 (1982); B212, 29 (1983).

[48] B. Dressler, M. Maul and C. Weiss, Nucl. Phys. B 578, 293 (2000).

[49] D. I. Diakonov, M. V. Polyakov and C. Weiss, Nucl. Phys. B461 (1996) 539. 
